ISAHP 1999, Kobe, Japan, August 12-14, 1999

\title{
INCORPORATING DATA INTO AHP MODELS
}

\author{
Ernest Forman and Mary Ann Selly \\ Department of Management Science, George Washington University \\ Washington D.C. 20052 \\ Expert Choice Inc. \\ Pittsburgh, PA. 15213 \\ Forman@gwu.edu http://mdm.gwu.edu/forman \\ Selly@expertchoice.com http://www.expertchoice.com
}

\begin{abstract}
Analytic Hierarchy Process (AHP) models can include data as well as judgments. This paper discusses several ways that data can be incorporated into AHP models. Data for alternatives with respect to covering objectives can be mapped into ratio scale preferences using linear increasing or decreasing functions, non-linear concave or convex increasing or decreasing functions, or step functions. Upper and lower bounds can also be specified over which these functions are defined. Qualitative data, such as ratings, can be mapped into a preference scale by making pairwise comparisons of the rating intensities. Data can also be applied in AHP models in two other contexts. First, as a screen to eliminate alternatives that do not satisfy one or more 'must' conditions, and second to derive priorities for covering objectives when all sibling covering objectives represent sub-dimensions of a well defined quantitative scale.
\end{abstract}

\section{Introduction}

Analytic Hierarchy Process (AHP) models, which typically process judgments, can also process data. The question arises as to what the various ways are of incorporating data in AHP models. This question is becoming increasingly more important as data becomes more abundant from sources such as the Internet and data warehouses.

In the following discussion, we will call the lowest level objectives in an AHP objectives hierarchy 'covering objectives' as they 'cover' the alternatives under consideration. Given data for a set of aiternatives with respect to a covering objective, preferences for the alternatives with respect to that covering objective can be obtained by through a process of pairwise comparisons based on the decision makers interpretation of the data, or by using some functional mapping, or formula. Each of these are addressed below.

\section{Pairwise Comparisons}

With one exception (discussed below) pairwise comparisons are always used to derive priorities of objectives or criteria in AHP models. Traditionally, the pairwise comparison process has also been used in the majority of AHP models to derive the priorities of alternatives. with respect to the lowest level subobjectives (henceforth called covering objectives) in the objectives hierarchy, and is the authors' preferred way of deriving priorities to reflect the decision maker's preferences for alternatives. This is the case regardless of whether or not data is available for the alternatives with respect to a given covering objective. When selecting a car, for example, there may be no data for 'appearance', but there typically is data about cost. When comparing the alternatives with respect to appearance, photographs can be used instead of data. Data, when available, serve as the basis of the decision maker's judgments about cost preference in the same way as photographs do for appearance preference. The data do not determine the priorities. Suppose the costs of the alternatives in a car selection model are $\$ 12,000, \$ 18,000, \$ 24,000$ 
and $\$ 42,000$. A decision maker, using the verbal AHP comparison mode, might judge the $\$ 12,000$ car to be strongly more preferable than the $\$ 18,000 \mathrm{car}$, strong-to-very strongly more preferable than the $\$ 24,000$ car, and so on. Priorities of the cars with respect to cost are then derived using the traditional right eigenvector calculation. Numerical and graphical pairwise comparisons can be made instead of using the verbal scale. Graphical comparisons more accurately represent the ratio of preferences of two alternatives than verbal comparisons and hence are recommended when time is a consideration and there is not enough time to make the full set of $n(n-1) / 2$ judgments for then elements being considered. In fact, a spanning set consisting of only n-1 pairwise comparisons (such as the diagonal elements of the comparison matrix) are required to calculate the priorities of $n$ alternatives. A few extra 'redundant' judgments can be included to increase accuracy. Still another efficient way to derive priorities using pairwise comparisons is to create small clusters with overlapping alternatives. Alternatives that appear in more than one cluster serve to link the clusters and determine a single ratio scale for the entire set of alternatives.

\section{Ratings Approach}

The ratings approach, which has long been used to derive priorities for a large number of alternatives, entails both pairwise comparisons and 'data'. Scales of 'rating intensities', such as 'Outstanding, Excellent, Very Good, Good, Fair, Poor' are defined and serve as surrogates for the alternatives. That is, instead of pairwise comparing the alternatives, the intensities are pairwise compared resulting in ratio scale priorities for the intensities. Each alternative is then rated using the defined intensities. In a sense, the rating intensity becomes data for the alternative, and the relative priority of the rating intensity becomes the relative priority of the alternative.

\section{Functional Mappings}

Although the interpretation of data by a decision maker using the pairwise comparison process is the preferred way of measuring preferences, a functional mapping or formula approach can be advantageous when there are a very large number of alternatives under consideration. There are, of course, no magic formulas for preference and it is important to keep in mind that the formula approach requires specific assumptions about the relationship between data and preference. These are described next.

\section{Linear and Nonlinear, Increasing and Decreasing Mappings}

Numerical data for alternatives with respect to the covering objectives in an AHP model can be mapped into ratio scale preferences for the alternatives using assumptions such as linear or non-linear concave or convex, increasing or decreasing functions. Upper and lower bounds can also be specified over which these functions are defined. A linear decreasing mapping, representing priorities for winter length (a subobjective of winter mildness in a model to evaluate retirement places (from Retirement Places Rated, Savageau[1995]) is shown in Figure 1, where priorities are represented by the length of the gray bars. The mapping represents Savageau's assumption that: "Winter length is scaled against a standard where 365 freezing days throughout the year gets a 0 , and no freezing days receives a perfect 100. " When this formula is applied to the number of freezing days for Ft. Meyers, Florida, and Bar Harbor, Maine, the ratio of their relative priorities as shown by the length of the bars in Figure 1, is less than 2 to 1. A result that may not fit many individuals relative preference. A convex mapping, such as represented in Figure 2, could be used instead - resulting in priorities shown in Figưre 3. Here, the ratio of preferences for Ft. Meyers over Bar Harbor (again shown by the relative lengths of the gray bars) is more than eight to one. Suffice it to say that, any mapping requires judgment and while there may be situations where there is general agreement on what is a reasonable mapping, it is important to not always take such mappings as a given, applicable to all subjects. 


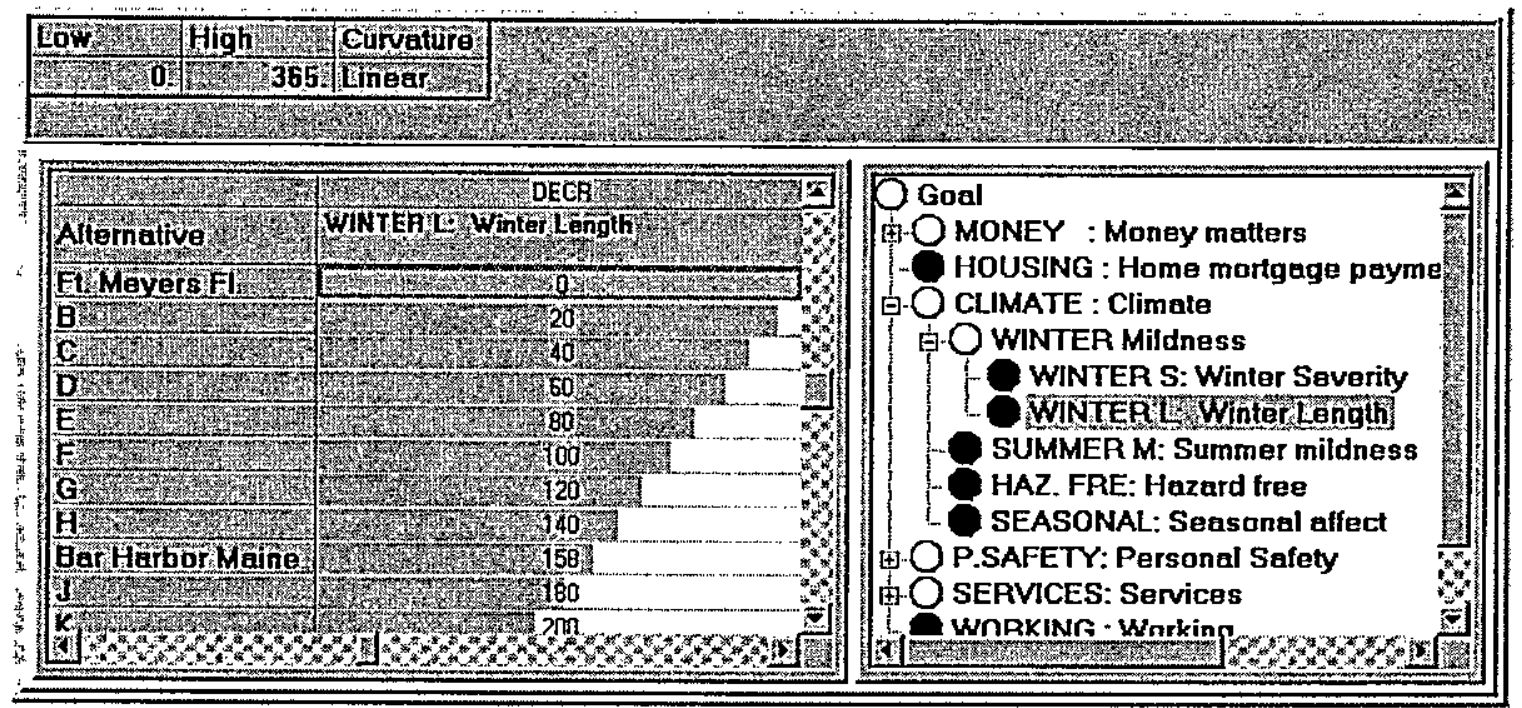

Figure 1 - Linear Decreasing Mapping

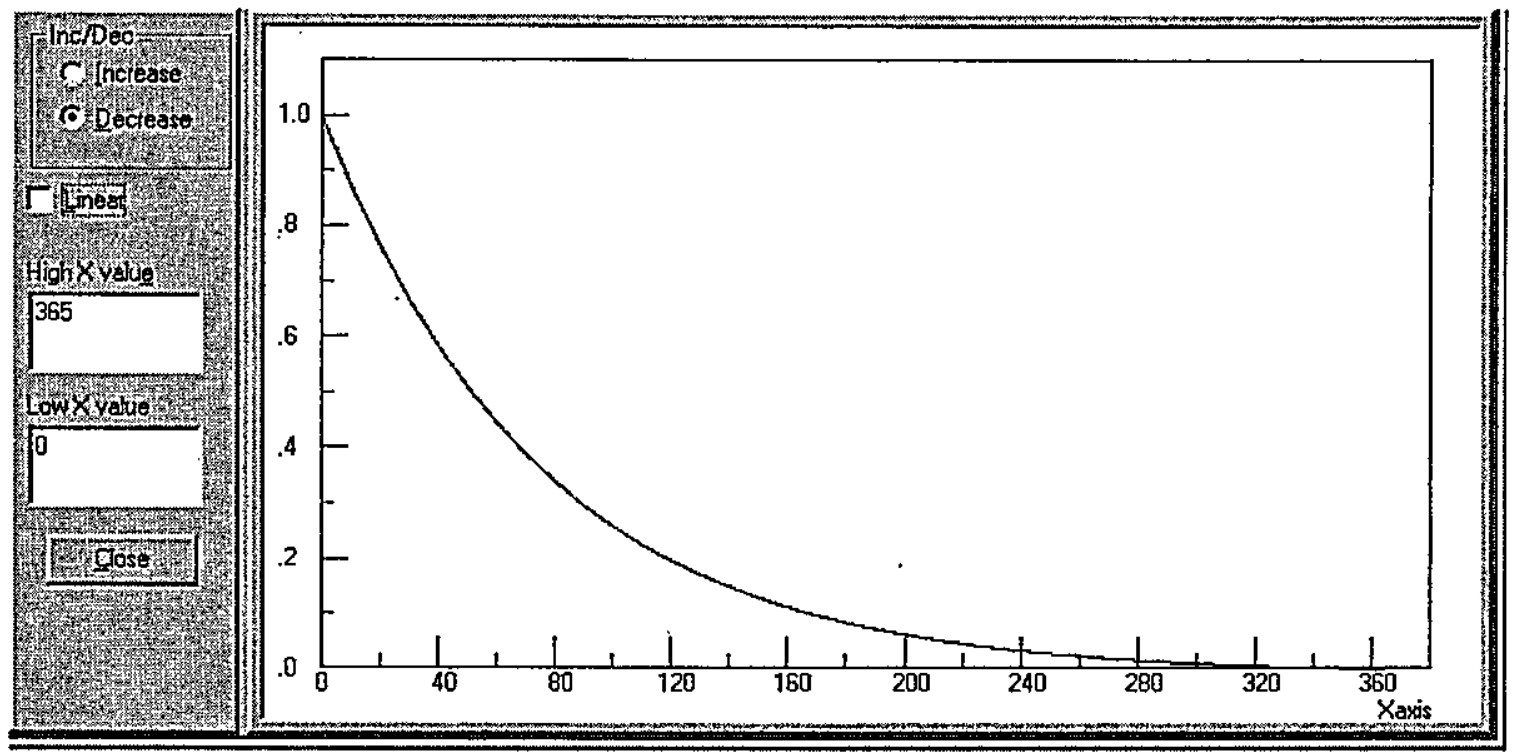

Figure 2 - Convex Decreasing Function

\section{Step Function Mapping}

A step function mapping is similar to rating alternatives using rating intensities, as discussed above, with the difference being that 1) step lower data bounds are defined for each of the intensities, and 2) data for an alternative, rather than judgment, determines which intensity applies. The priorities for the intensities can be specified directly, or derived through pairwise comparisons. For example, in rating the preference of cities with respect to the number of people per public hole golf course - $\mathrm{a}$ ' $A A^{\text {' }}$ rating applies if there are fewer than 800 people per hole, an ' $\mathrm{A}$ ' rating if there are more fewer than 1350 people per hole, and so on; see Figure 4.

The priorities shown in Figure 4 have been set according to Retirement Places Rated, but could been derived by pairwise comparing the relative preferences of the steps. 


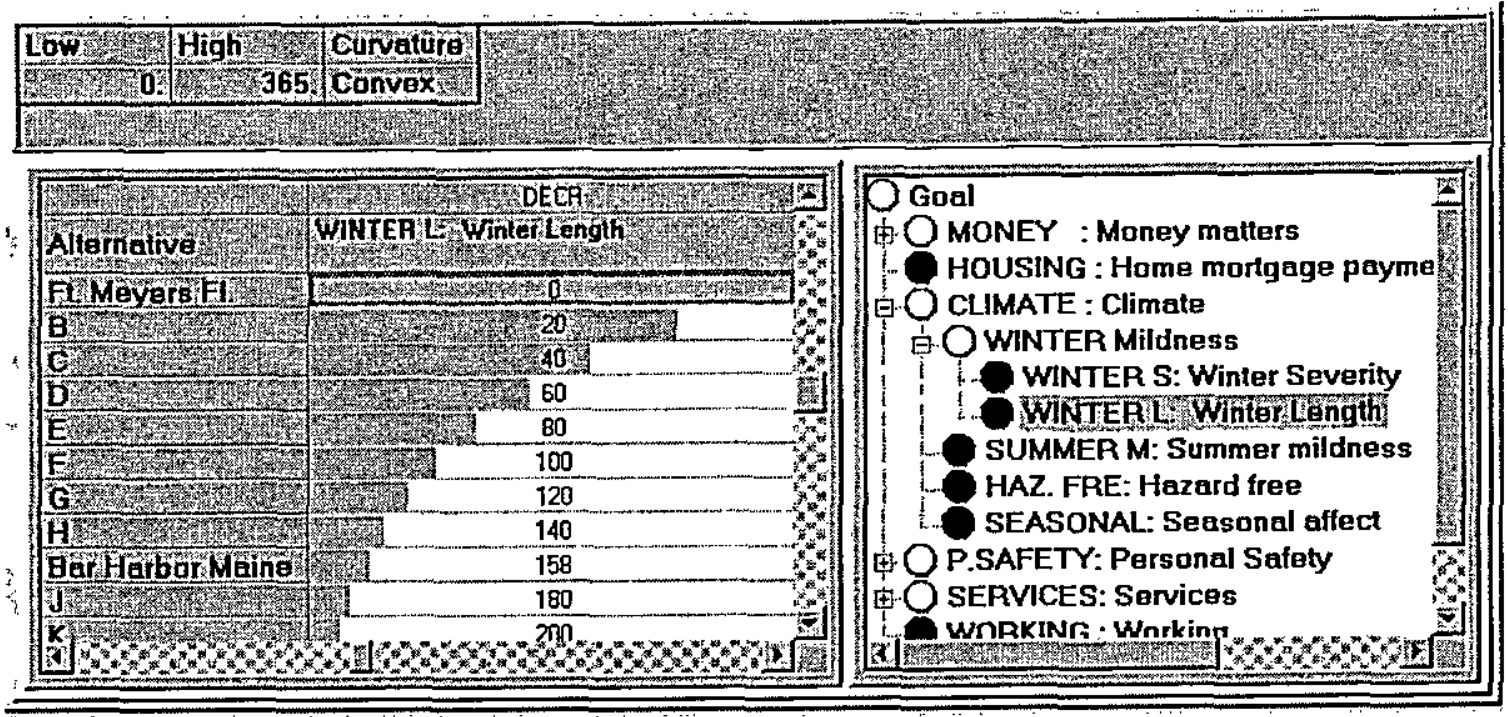

Figure 3 - Priorities resulting from the Convex Decreasing Mapping

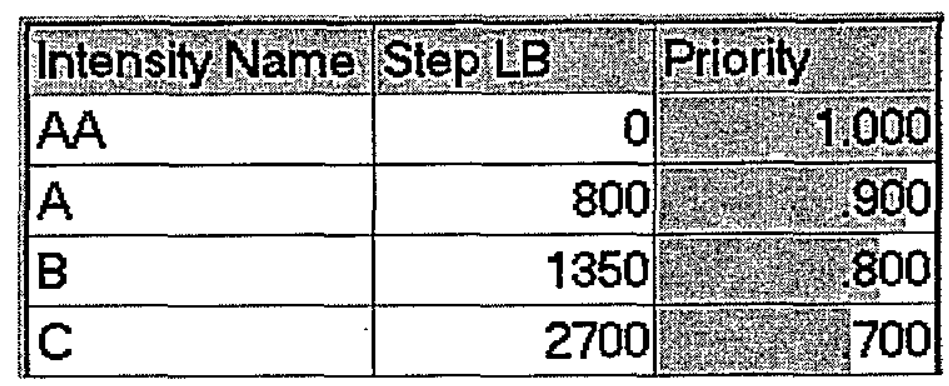

Figure 4 -Step function Mapping

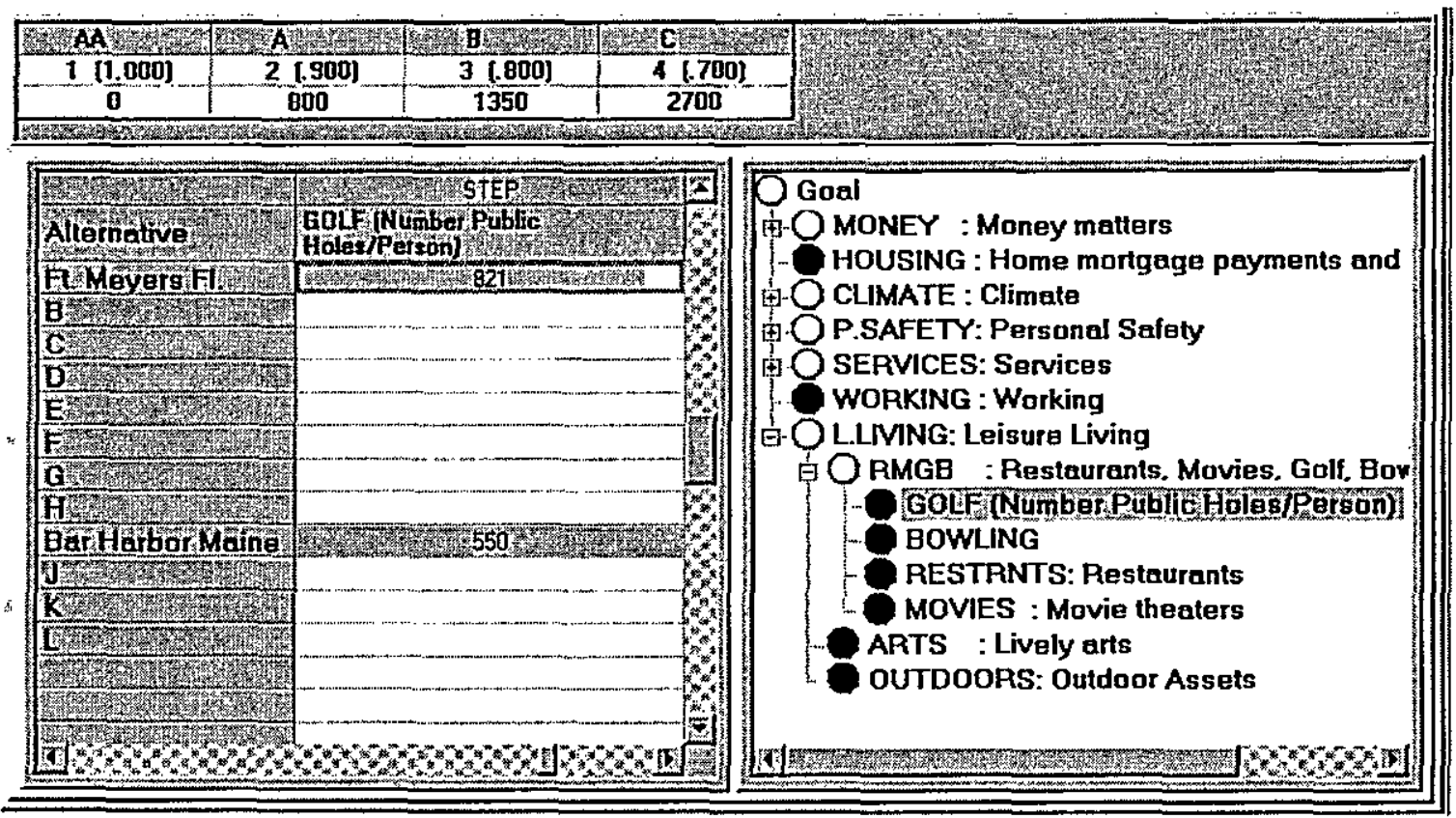

Figure 5 - Priorities resulting form Step Function Mapping 


\section{Feasible Alternatives - Musts}

In addition to using data to derive preferences for alternatives with respect to their covering objectives, data can also be used in two other contexts in AHP models. While AHP is a linear compensatory decision theory that uses measures on a ratio scale, data is sometimes used as an ordinal measure to include or exclude alternatives from considerations. In other words, an AHP model helps measure what we 'want' to have, while the ordinal use of data to screen alternatives is a way of saying what we 'must' have. Theoretically, the value of any must, is, in almost all cases, arbitrary and it seldom makes sense to include an alternative with a value just slightly above some value while excluding another just slightly below that value. In practice however, the use of data to screen alternatives can reduce the number of alternatives under consideration considerably, so that a conservative selection for the 'must' values will help in finding the best alternative(s) without incurring a significant danger of eliminating desirable alternatives. An example of using musts to screen alternatives is shown in Figure 6, where it is specified that there be at most 700 people per public course golf hole.

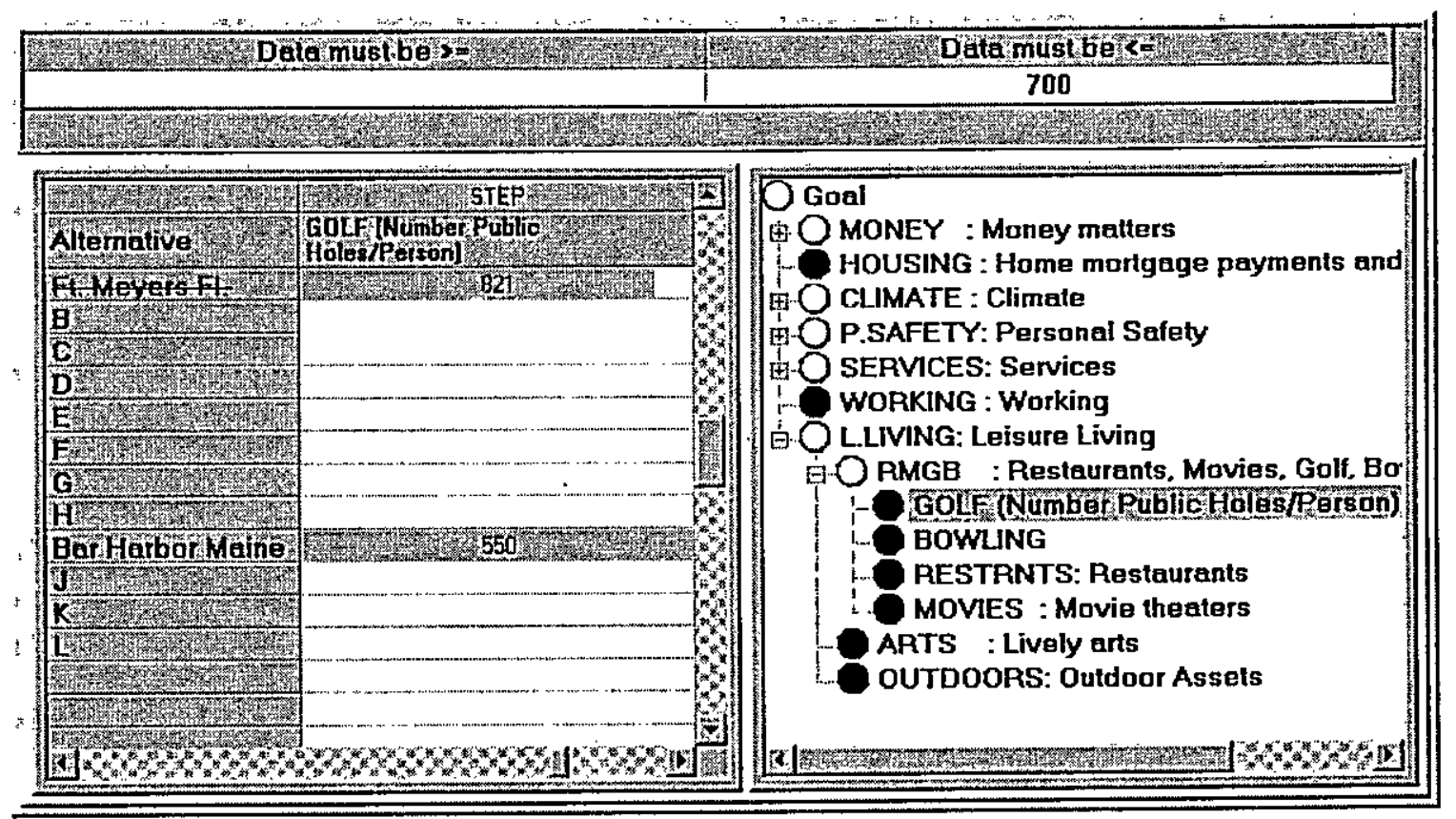

Figure 6 - Must Screen Showing Infeasible Alternatives

\section{Data Determination of Covering Objective Importance}

Finally, data for the alternatives, can, in some special cases, be used to derive priorities for covering objectives when all sibling covering objectives represent sub-dimensions of a well defined quantitative scale. This makes sense only when the covering objectives represent divisions of a common scale (such as perimeter being divided into length and width) where simple algebra or a spreadsheet calculation could be used to combine the covering objectives. This is illustrated below with an example taken from Schenkerman [1997] where the perimeters of four fields A, B, C and D (shown in Figure 7) are to be prioritized. First the data for the alternatives can be used to prioritize the alternatives with respect to the covering objectives - length and width. This is shown by the lengths of the gray bars in Figure 7. Second, the priorities of the covering objectives can also be derived from the sum of the data under each covering objective (10 and 30 respectively). See Figure 8 . The synthesis of this mode results in the alternative priorities shown in Figure 9. 


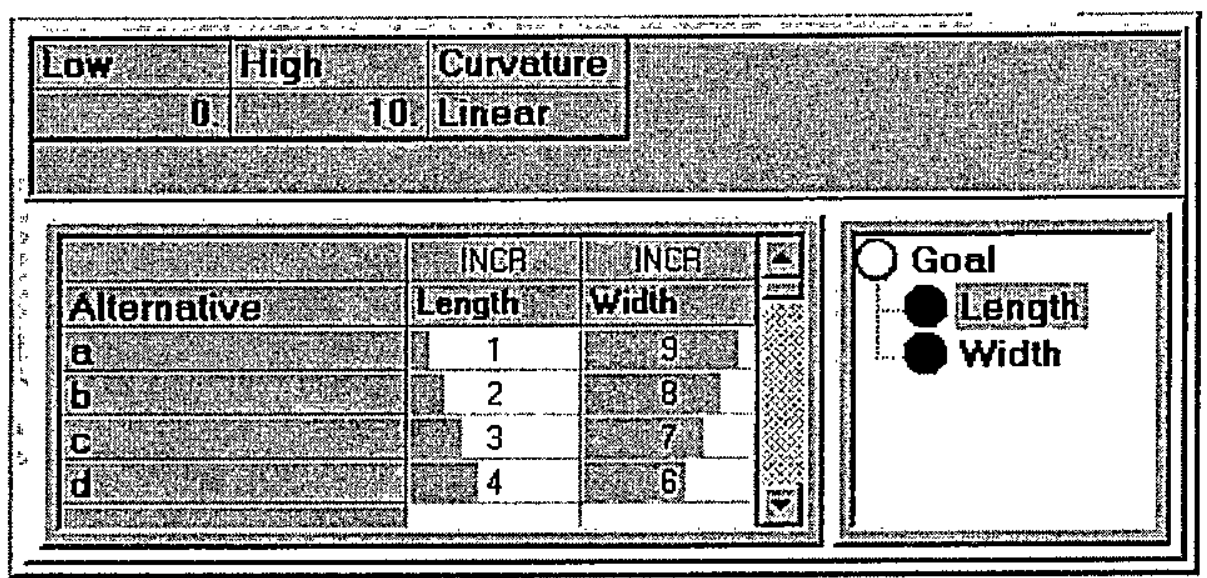

Figure 7-Priorities of the Alternatives with respect to Length and Width

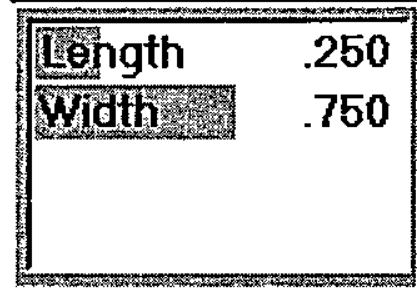

Figure 8 - Covering Objectives' Priorities

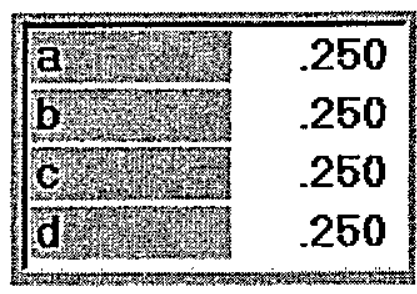

Figure 9-Synthesis Results

We note that the algebraic derivation of priorities of objectives from alternative data does not make sense for the majority of AHP applications where judgment is required to assess the relative importance of disparate covering objectives such as cost, comfort and performance.

\section{Summary}

The ability to incorporate subjective judgments sets AHP apart from most other modeling approaches. But that does not preclude the incorporation of data into AHP models as well. Qualitative data in the form of rating intensities, such as 'Excellent, Very Good, Good, Fair, Poor' can be mapped into a preference scale by pairwise comparing the rating intensities. Data for alternatives with respect to covering objectives can be mapped into ratio scale preferences using linear or non-linear, convex or concave functions. Step functions can transform data into rating intensities, which are themselves prioritized with pairwise comparisons. Data can also be used to screen or eliminate alternatives that do not satisfy one or more 'must' conditions. Finally, in some very special cases, data can be used to derive priorities for covering objectives when all sibling covering objectives represent sub-dimensions of a well defined quantitative scale.

\section{References}

Forman, E. H. (1999), Expert Choice Version 10, Expert Choice Inc., Pittsburgh, PA.

Schenkerman, S. (1997), Inducement of Nonexistent Order by the Analytic Hierarchy Process, Decision Sciences, 28 (2), 475-482. 